\title{
2-Aminobenzamide and 2-Aminobenzoic Acid as New MALDI Matrices Inducing Radical Mediated In-Source Decay of Peptides and Proteins
}

\author{
Nicolas Smargiasso, Loic Quinton, Edwin De Pauw
}

Mass Spectrometry Laboratory, GIGA-Research, University of Liege, Allee de la Chimie, 3, BAT B6c, 4000 Liege, Belgium

\begin{abstract}
One of the mechanisms leading to MALDI in-source decay (MALDI ISD) is the transfer of hydrogen radicals to analytes upon laser irradiation. Analytes such as peptides or proteins may undergo ISD and this method can therefore be exploited for top-down sequencing. When performed on peptides, radical-induced ISD results in production of c- and z-ions, as also found in ETD and ECD activation. Here, we describe two new compounds which, when used as MALDI matrices, are able to efficiently induce ISD of peptides and proteins: 2-aminobenzamide and 2-aminobenzoic acid. In-source reduction of the disulfide bridge containing peptide Calcitonin further confirmed the radicalar mechanism of the ISD process. ISD of peptides led, in addition to $\mathrm{c}$ - and $\mathrm{z}$-ions, to the generation of a-, $\mathrm{x}$-, and $\mathrm{y}$-ions both in positive and in negative ion modes. Finally, good sequence coverage was obtained for the sequencing of myoglobin (17 kDa protein), confirming the effectiveness of both 2-aminobenzamide and 2-aminobenzoic acid as MALDI ISD matrices.
\end{abstract}

Key words: MALDI, In-Source decay, Fragmentation, Peptides, Proteins

\section{Introduction}

$\mathrm{M}$ ALDI in-source decay (or MALDI ISD) has been observed for various kinds of analytes such as peptides and proteins [1-6], carbohydrates [7-12] oligonucleotides [13], and is currently used for top-down protein sequencing [14]. Concerning peptides and proteins, two main activation modes, thermal and radical-mediated, were described in literature to explain the in-source fragmentation pathways. The thermal activation leads to the formation of

Electronic supplementary material The online version of this article (doi:10.1007/s13361-011-0307-5) contains supplementary material, which is available to authorized users.

Correspondence to: Nicolas Smargiasso; e-mail: nsmargiasso@ulg.ac.be
"CID-like" b- and y-fragments, essentially created via collisions during the desorption step of analytes and the expansion of the MALDI plume [15-17]. However, a radical-mediated mechanism was proposed by Takayama et al. to explain the presence of c- and z-ions observed in MALDI-ISD spectra of peptides and proteins. In this model, a hydrogen radical is first transferred from the MALDI matrix to a carbonyl group of a peptide bond during laser irradiation. This leads to the formation of a carbon-centered radical that is structurally similar to those found during ECD fragmentation $[18,19]$. It undergoes further fragmentation at the $\mathrm{NH}-\mathrm{C} \alpha$ bond. This model was then completed by Demeure et al. in order to explain the occurrence of d- and w-ions [17].

As thermal activation allows a redistribution of the internal energy received by the analyte, it is not suitable for top-down sequencing of large proteins that have a high 
number of degrees of freedom. The radical-mediated pathway overcomes this limitation, as the redistribution of internal energy of the analyte is not the driving force of the reaction. This pathway would not be theoretically limited by the mass of the analyte, thus allowing ISD to be used to sequence large proteins. Moreover, it was demonstrated that during radical-induced ISD and just like ETD or ECD, posttranslational modifications are preserved, including labile ones such as phosphorylation [19-21]. Nevertheless, since radical-mediated ISD is a reductive reaction, disulfide bonds could be altered [22], and information concerning their position could be lost. On the other hand, this reductive property was used to identify disulfide-linked peptides [23, 24] or to classify cyclical peptides, such as microcystins, which contain modified amino acids (such as dehydroalanine) that are also susceptible to being reduced upon ISD [25].

The MALDI matrices 2,5-dihydroxybenzoic acid (2,5DHB) [2], 5-aminosalycilic acid (5-ASA) [3], and 1,5diaminonaphthalene $(1,5-\mathrm{DAN})[4,26]$ were previously described to efficiently induce radical mediated ISD. Among them, 1,5-DAN is the most efficient due to its high radical transfer ability, but it promotes the formation of intense matrix cluster ion peaks, potentially interfering with the detection of analyte fragment peaks [17]. More recently, two additional matrices, 5-nitrosalicylic acid (5-NSA) and 5formylsalicylic acid (5-FSA) that lead to the formation of a- and $\mathrm{x}$-ions were described by Asakawa and Takayama [27]. In this case, a transfer of hydrogen from a $\mathrm{NH}$ group of a peptide bond to the matrix would occur, leading to the formation of a nitrogen-centered radical. This radical would subsequently induce the fragmentation of the $\mathrm{C} \alpha-\mathrm{C}$ bond.

Here, we report the ability of two new MALDI matrices to produce radical-induced ISD: 2-aminobenzamide (2-AB) and 2-aminobenzoic acid (2-AA). These matrices are chemically stable under normal laboratory conditions, very soluble, and nontoxic compounds. As 1,5-DAN is an unstable carcinogenic compound [4], these new easy-to-use matrices could become useful alternatives to previously described ISD matrices.

\section{Experimental}

\section{Materials}

2-Aminobenzamide (2-AB), 2-aminobenzoic acid (2-AA) and 1,5-diaminonaphthalene (1,5-DAN) were purchased from Sigma-Aldrich (Steinheim, Germany). The peptides calcitonin (salmon I), (Tyr $\left.{ }^{0}\right)$-C-peptide (dog), (Glu $\left.{ }^{1}\right)$ fibrinopeptide B, acetyl-amylin were purchased from Bachem (Weil am Rhein, Germany). All the solvents used were HPLC grade quality. The proteins myoglobin and human serum albumin (HSA) were purchased from Sigma-Aldrich (Steinheim, Germany).

\section{MALDI Spots Preparation}

All peptides (Table 1) were prepared at $20 \mu \mathrm{M}$ in water. Stock solutions of myoglobin and HSA were prepared at $1 \mathrm{mg} /$ $\mathrm{mL}$ and were further diluted to spot 200 pmol on the MALDI plate.

For matrices, 2-AA and 2-AB solutions were both prepared in $\mathrm{H}_{2} \mathrm{O} / \mathrm{ACN}(1 / 1), 0.1 \%$ formic acid at $20 \mathrm{mg} / \mathrm{mL}$. 1,5-DAN solutions were prepared at saturation in $\mathrm{H}_{2} \mathrm{O}$ / ACN (1/1), $0.1 \%$ formic acid shortly before use due to its instability.

All spots were prepared by mixing equivolumes of matrix and analyte following the "dried droplet" method. Spots prepared with both 2-AA and 2-AB as the MALDI matrix are not homogeneous and analytes are located in needleshaped crystals and in the rim of the spots (Figure S-1). 2$\mathrm{AB}$ spots were found to be stable under vacuum while 2-AA spots degraded slowly under these conditions, therefore limiting the available time for spot analysis. Both spots were stable at atmospheric pressure and room temperature and analytes were not degraded by these conditions, thus allowing preservation of spots for further analysis.

\section{Mass Spectrometry}

Spectra were recorded on a Bruker Ultraflex II TOF/TOF, controlled by the FlexControl 3.0 software (Bruker Daltonics, Bremen, Germany). Desorption/Ionization was performed using a frequency tripled Nd:YAG laser emitting at $355 \mathrm{~nm}$ with a $100 \mathrm{~Hz}$ shot frequency. Variable laser power was used according to the MALDI matrix: roughly, 2-AB needs a higher laser power than 2-AA while the laser power used for 2-AA is similar to that used for 2,5-DHB. Concerning spectra of peptides, 1000 laser shots were acquired for experiments on calcitonin and $\left(\mathrm{Glu}^{1}\right)$-fibrinopeptide B and 5000 shots for experiments on acetyl-amylin and $\left(\mathrm{Tyr}^{\mathrm{O}}\right)$-C-peptide. For proteins, 10,000 shots were summed. The spectrometer was tuned with the following parameters: $25 \mathrm{kV}$ acceleration voltage, $21.85 \mathrm{kV}$ pulse voltage, $30 \mathrm{~ns}$ ion extraction delay, and $1.4 \mathrm{kV}$ detector gain. FlexAnalysis 3.0 and Biotools 3.2

Table 1. Name, Sequence and Masses of Peptides Used in this Study

\begin{tabular}{lll}
\hline Name & Sequence & Monoisotopic mass \\
\hline$\left(\right.$ Glu $\left.^{1}\right)$-fibrinopeptide B & EGVNDNEEGFFSAR & 1569.7 \\
$\left(\mathrm{Tyr}^{0}\right)$-C-peptide $(\mathrm{dog})$ & YEVEDLVRDVELAGAPGEGGLQPLALEGALQ & 3335.7 \\
Calcitonin & CSNLSTCVLGKLSQELHKLQTYPTRTNTGSGTP CONH & 3 \\
acetyl-amylin & Ac-ATQRLANFLVHSSNNFGAILSSTNVGSNTY-CONH & 3431.7 \\
\hline
\end{tabular}


softwares were used to analyze and annotate spectra (Bruker Daltonics, Bremen, Germany).

\section{Results and Discussion}

\section{Evaluation of the Hydrogen Radical Transfer Ability}

As mentioned above, radical mediated MALDI-ISD is a reductive process that potentially leads to the cleavage of disulfide bonds. This property can be exploited as a probe of ISD efficiency [3, 17] and the in-source reduction of salmon calcitonin was tested. Salmon calcitonin is a peptide containing one intrapeptidic disulfide bond and its reduction can be easily determined after deconvoluting of the experimental isotopic pattern. Briefly, based on the first two peaks of the distribution, the area of the peaks belonging to the oxidized form was calculated and then subtracted from experimental peak distributions, allowing calculation of the area of the peaks belonging to the reduced form. Spectra obtained for 2-AA and 2-AB are shown in Figure 1. The proportion of reduced calcitonin was $27 \% \pm 2 \%$ for 2 $\mathrm{AA}$ and $44 \% \pm 4 \%$ for 2 - $\mathrm{AB}$ ( 15 spectra from three spots were recorded for each condition). Compared with values obtained for other known ISD inducing matrices such as DHB (21\%) or DAN (84\%) [17], these proportions of the reduced form are intermediate, confirming that these molecules could be used as efficient ISD-inducing matrices.

\section{ISD of Peptides in Positive Ion Mode}

It was shown previously that, according to MALDI matrix properties, different type of ions could be produced upon ISD of peptides. In order to determine which fragments are produced during ISD using 2-AA and 2-AB, the peptides ( $\left.\mathrm{Glu}^{1}\right)$ fibrinopeptide $\mathrm{B},\left(\mathrm{Tyr}^{\mathrm{O}}\right)$-C-peptide ( $\left.\mathrm{dog}\right)$, and acetyl-amylin were chosen as test models. Indeed, $\left(\mathrm{Glu}^{1}\right)$-fibrinopeptide B contains an arginine (easily protonated) in the C-terminal part, which leads to the preferential observation of ions containing this part (i.e., $\mathrm{x}-$, $\mathrm{y}$ - and $\mathrm{z}$-type ions) in positive ion mode. Inversely, $\left(\mathrm{Tyr}^{0}\right)$-C-peptide and acetyl-amylin contain an arginine in the N-terminal part, leading to the preferential formation of a-, b-, and c-type ions [17, 28]. Ions are named here according to the Zubarev et al. nomenclature [29].

Results obtained for $\left(\mathrm{Glu}^{1}\right)$-fibrinopeptide B (Figure S-2) show that the most abundant fragments are $y-$ and $z^{\prime}$-ions. The presence of z-type ions confirms the activation by the radical-mediated fragmentation pathway. Besides intense $y-$ and $\mathrm{z}$-ions, $\mathrm{x}$-ions were also observed using these two matrices (Figure S-2B). As described by Takayama et al., these ions would result from the fragmentation of an $\mathrm{N}$ centered radical produced after the transfer of a hydrogen radical from the peptide NH group onto the matrix. This would indicate that 2-AA and 2-AB are both able to accept

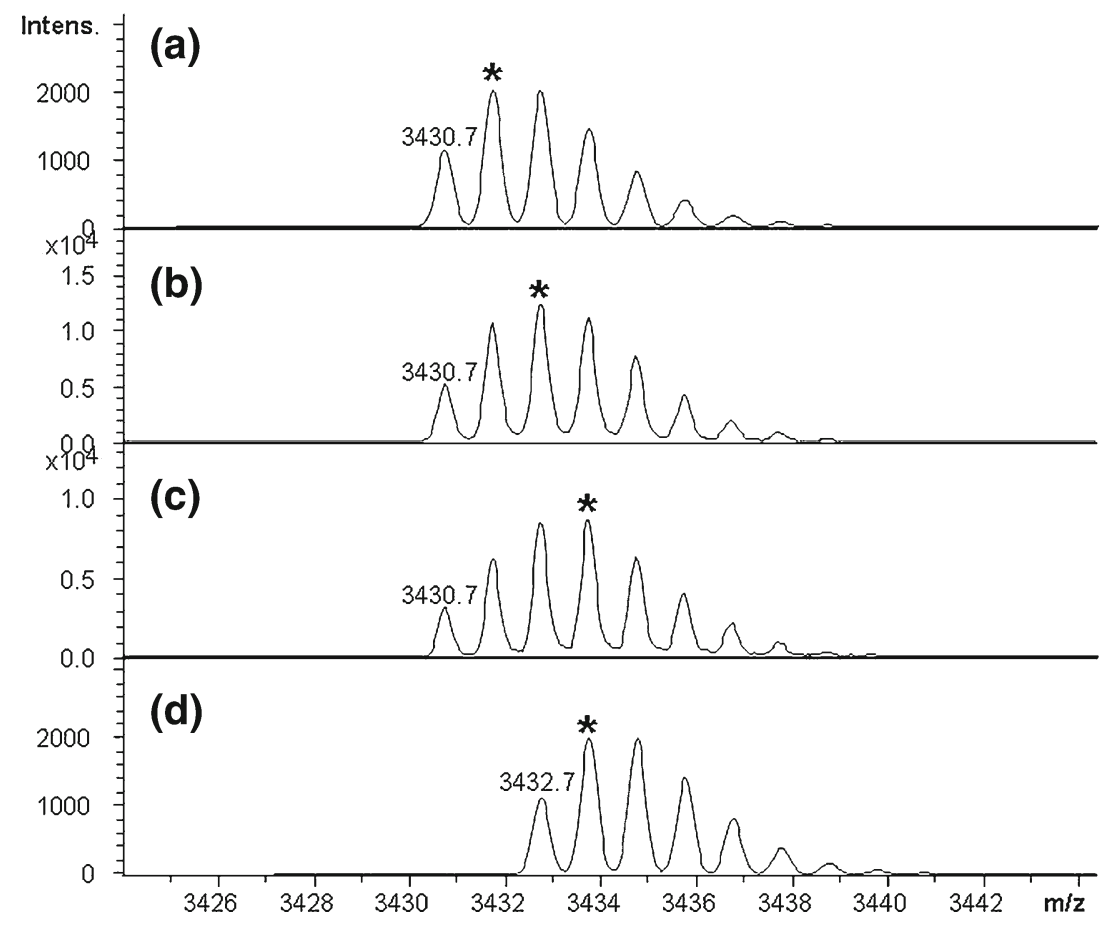

Figure 1. (a) Theoretical spectrum of the oxidized calcitonin; (b), (c) experimental spectra obtained with 2-AA and 2-AB, respectively; (d) theoretical spectrum of the reduced calcitonin. The most intense peak of each spectrum is marked with an asterisk (*) 
and transfer $\mathrm{H}$ radicals. This behavior was also previously described for 5-formylsalicylic acid (5-FSA) [27]. Moreover, $\mathrm{x}^{\prime}$-ions are present, indicating either a reduction of $\mathrm{x}$-ions, or the capture of a hydrogen radical by $\mathrm{x} \cdot$ ions that would be formed after the fragmentation of the $\mathrm{C}_{\alpha}-\mathrm{C}$ bond. Nevertheless, the pathway leading to the formation of the $\mathrm{a} / \mathrm{x} \cdot$ pair was showed to be unfavored when 5-NSA or 5-FSA are used, indicating possible differences compared with fragmentation pathways induced by $2-\mathrm{AB}$ and 2-AA.

The presence of w-ions was previously described as produced by ISD by fragmentation of $z^{\prime}$-ions using the DAN matrix [17]. Interestingly, $2-\mathrm{AA}$ and $2-\mathrm{AB}$ promote the formation of w-ions, but also reduced w-ions (Figure S-2C) and $\mathrm{v}$-ions, possibly resulting from the degradation of $\mathrm{y}$ - or $\mathrm{x} \cdot$ ions [30] (Figure S-2D). However, the ratios between $\mathrm{w}$ - and $\mathrm{v}$ ions are different between 2-AA and in 2-AB, indicating possible matrix related differences in the activation pathways of the first generation of ions during the ISD process.

As expected, fragmentation of acetyl-amylin (Figure 2) and $\left(\mathrm{Tyr}^{0}\right)$-C-peptide (Figure S-3) show a predominant formation of $\mathrm{c}^{\prime}$-ions, and the whole sequence was covered, with both matrices. In addition to $\mathrm{c}^{\prime}$-ions, a-type ions are also present in the spectra. It appears that for a given MALDI matrix, areas of a-type ions vary greatly according to the residue and its position, in comparison to the areas of the related c-ions. However, 2-AB produces systematically more a-ions than 2-AA, while DAN produces only a small amount of a-ions (area of a-ions is generally under $10 \%$ of related c-ions, data not shown). In Figure $2 \mathrm{~b}$ and $\mathrm{c}$, it appears that these a-type ions are in fact a mixture of a-, a $\bullet^{-}$, and $\mathrm{a}^{\prime}$-ions, indicating the probable involvement of several fragmentation pathways in the formation of these ions. Moreover, depending on the MALDI matrix used and the nature of the amino acid residue, proportions of different atype ions vary (Table S-1 and S-2). Accordingly, it can be seen that 2-AA tends to produce a higher proportion of aions than does 2-AB. Indeed, the proportions of a-ions obtained with 2-AA are always higher than $50 \%$, while this value varies from 25 to $50 \%$ for $2-\mathrm{AB}$. Consequently, both $\mathrm{a}^{\bullet}$ and $\mathrm{a}^{\prime}$ proportions are generally increased when $2-\mathrm{AB}$ is

(a)
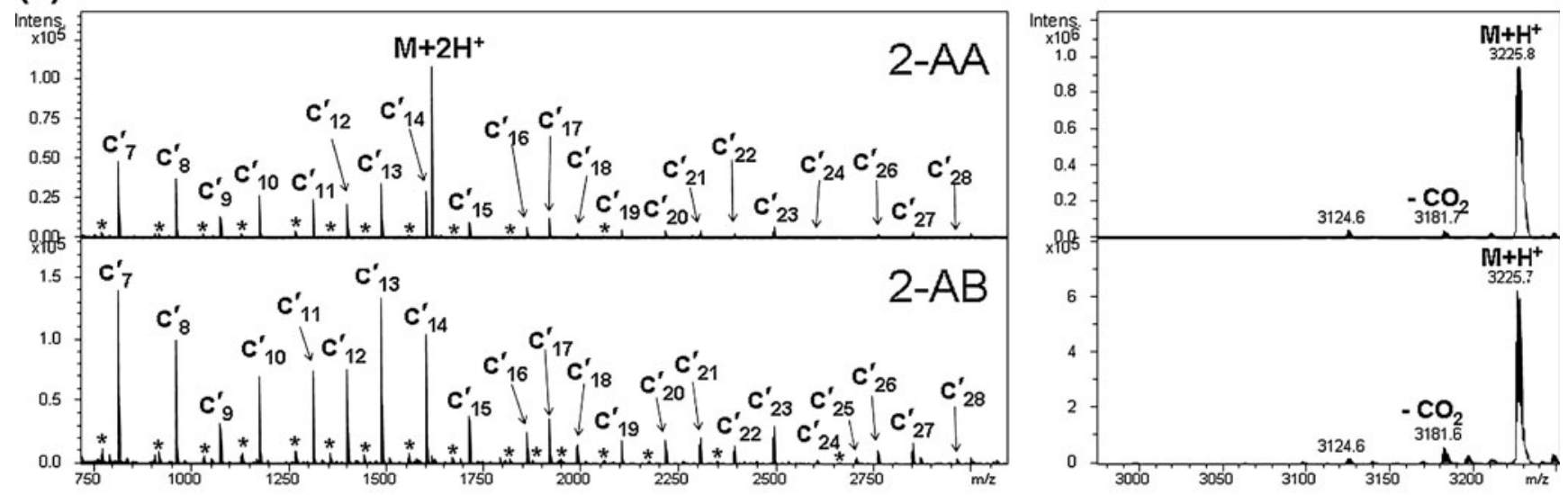

(b)

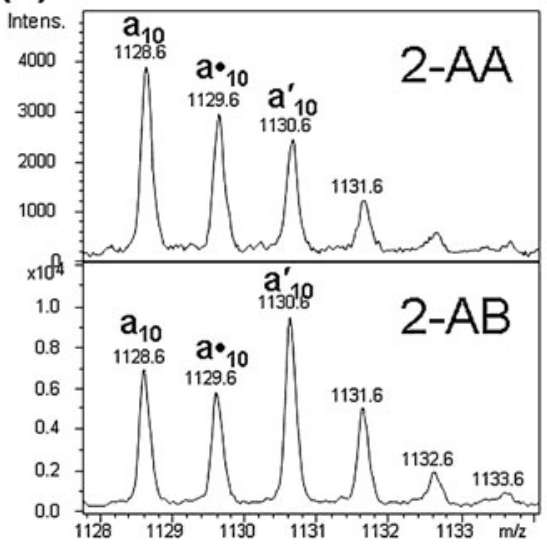

(c)

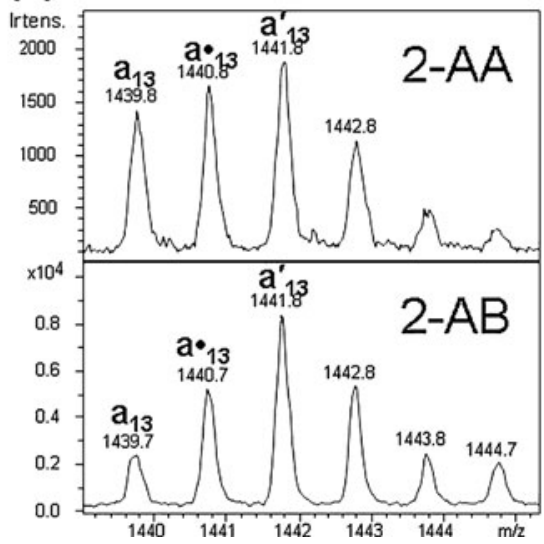

Figure 2. (a) Spectrum obtained for acetyl-amylin in positive ion mode with 2-AA as the matrix and 2-AB. Peaks marked by an asterisk indicate a-type ions. (b) Zoom on the region containing the fragments $a_{10}(\mathrm{Val})$. The most intense ion is the $\mathrm{a}_{10}$ with 2 AA while $a^{\prime}{ }_{10}$ is dominant with 2-AB (proportions of $a$, a and $a^{\prime}$-ions are 71,7 , and $22 \%$, respectively, with 2 -AA as matrix and $41 \%, 13 \%$, and $46 \%$, respectively, with $2-A B$ as matrix). (c) Zoom on the region containing the fragment $\mathrm{a}_{13}$ (Ser- proportions of $\mathrm{a}, \mathrm{a} \cdot$, and $\mathrm{a}^{\prime}$-ions are 51,12 , and 37 , respectively, with $2-\mathrm{AA}$ as matrix and 23,30 , and 47 , respectively with 2 -AB as matrix) 
used. On the other hand, important variations are observed according to the amino acid residue. Indeed, it can be seen that residues containing aliphatic side chains preferentially promote the formation of a-ions (Figure 2b) while other residues tend to produce more $\mathrm{a}^{\bullet-}$ and $\mathrm{a}^{\prime}$ - ions. This is the case for serine residues (Figure 2c). It should be noted that glycine residues induce the preferential formation of $\mathrm{a}^{\prime}$-ions (Figure S-3B), while glutamic acid residues favor the formation of "a-like ions", in which the loss of $45 \mathrm{Da}$ could be due to a fragmentation of the side chain, in good agreement with previous observations reported by Takayama et al. (Figure S-3C). Concerning the origin of these different a-type ions, it was proposed that a and $\mathrm{a}^{\prime}$ ions result from thermal activation of c-ions. Our results show that 1,5-DAN and $2-\mathrm{AB}$ produce more $\mathrm{a}^{\prime}$ ions than 2-AA (except when the residue is glycine), indicating a possible involvement of the hydrogen radical transfer ability of the matrix in the production of $\mathrm{a}^{\prime}$ ions. Concerning a $\boldsymbol{a}^{\bullet}$ ions, several fragmentation pathways could lead to the production of this radical species: (1) a $a$ ions were previously described as intermediates species during the rearrangement of $c^{\bullet}$ ions into d-ions $[17,30,31]$. Some d-ions were observed in experimental spectra of $\left(\mathrm{Tyr}^{0}\right)$-C-peptide, confirming that these reactions may occur with 2-AA and 2-AB as matrices (2) a• ions could be formed during a homolytic cleavage of the $\mathrm{C} \alpha-\mathrm{C}$ bond leading to the formation of $\mathrm{x}$-ions. This pathway is supported by the observation of $\mathrm{x}$-ions during ISD of ( $\left.\mathrm{Glu}^{1}\right)$ fibrinopeptide B. Moreover, as mentioned previously, it was described by Takayama et al. that the production of $\mathrm{a} \bullet \mathrm{x}$ ion pairs was favored over the production of $\mathrm{a} / \mathrm{x} \cdot$ during the cleavage of $\mathrm{C} \alpha-\mathrm{C}$ bonds [27] (3) a• ions were described as an alternative (and minor) product in ECD that leads to the formation of $a^{\bullet}$ and y-ions [32]. As ions involved in these three pathways (d-, $\mathrm{x}$ - and $\mathrm{y}$-ions) were found in our spectra, it is not possible to determine if all three or only one of them are involved in the production of $a \cdot$ ions. This point will need future investigation.

\section{ISD of Peptides in Negative Ion Mode}

As it is difficult to observe acidic peptides in positive ion mode due to the lack of proton acceptor sites, they can be analyzed in negative ion mode. To ensure that 2$\mathrm{AA}$ and $2-\mathrm{AB}$ are able to induce ISD in negative ion mode, we decided to use $\left(\mathrm{Glu}^{1}\right)$-fibrinopeptide $\mathrm{B}$ as model peptide because this peptide possess four acidic residues. Obtained spectra (Figure S-4) show that both 2$\mathrm{AA}$ and $2-\mathrm{AB}$ are efficient matrices in negative ion mode. Moreover, ISD was also observed in this mode, emphasizing the versatility of these new matrices. The fragments that were observed in negative ion modes are similar to those that were found in positive ion mode. Of course, the negative ion mode allowed observing both $\mathrm{N}$ terminal (a- and c-ions) and C-terminal (x-, y- and z-ions) of $\left(\mathrm{Glu}^{1}\right)$-fibrinopeptide $\mathrm{B}$, due to the presence of several potential negatively charged sites.

\section{ISD of Proteins with 2-AA or 2-AB as the Matrix}

To evaluate the ability of 2-AA and 2-AB to be used for topdown sequencing of proteins, ISD was tested on myoglobin $(\mathrm{Mw} \approx 17 \mathrm{kDa})$ and oxidized HSA (Human serum albumin, $\mathrm{Mw} \approx 66 \mathrm{kDa}$ ). For both proteins, the limit at which ISD was no longer observed was around 10-20 pmol per spot for 2AA, and 50-60 pmol per spot for 2-AB (data not shown). For myoglobin (Figure S-5), sequence coverages of more than $60 \%$ were obtained for both 2-AA and 2-AB. These coverage values are similar to those obtained with the 1,5DAN matrix. However, the accumulation of more spectra was needed with 2-AA and 2-AB as matrices than for 1,5DAN to obtain workable spectra for sequencing purpose. This is probably due to the lower efficiency of these two compounds in inducing hydrogen radical transfer. As expected, numerous a- and x-type ions were produced, allowing the sequencing to overcome the presence of proline gaps. Concerning HSA (Figure S-6), its oxidized form was used in order to evaluate the ability of the matrices to sequence proteins containing several disulfide bonds [17]. In Figure S-6, it can be seen that practically only c-ions are present in the spectra, whichever the matrix (2-AA or 2-AB) is used. This may be rationalized by the presence of several cysteines located on the C-terminal part of the sequence, implying that the disulfide bonds in which these cysteines are involved are preferentially reduced during ISD, thus impeding the sequencing. The obtained sequence covers the 30 first amino acids in the case of 2-AA, which is similar to the results obtained with 1,5 -DAN on the same protein (data not shown), while a smaller sequence coverage was obtained for 2-AB. Interestingly, $\mathrm{c}^{\prime}{ }_{12}$ is the most intense fragment with both matrices and the $\mathrm{c}_{22}^{\prime}$ ion is more intense compared with its neighbors, with 2-AA as matrix, indicating preferential fragmentation sites. In addition, with both 2$\mathrm{AA}$ and $2-\mathrm{AB}$, the intensity of the $\mathrm{c}_{33}$ ion is relatively important, as this residue is located before the first cysteine of the protein. After that residue, ISD peaks are not detected anymore, probably due to the preferential reduction of disulfide bonds, potentially impeding sequencing.

\section{Conclusions}

2-AA and 2-AB, two molecules that had never been described as potential MALDI matrices for analysis of peptides and proteins, were described here as suitable matrices for MALDI-ISD. Besides production of c- and zions, it was shown that 2-AA and 2-AB are able to produce different other types of ions, including a-, $\mathrm{x}-$, and v-ions. These observations raise questions about the exact mechanisms governing the formation of these ion types and the potential differences existing between ISD fragmentation induced by well-established matrices like 1,5-DAN or 2,5DHB and these new matrices. As an important difference in the hydrogen radical transfer ability was observed between these two molecules, this implies that others factors 
(crystallization, ability to ionize, and/or desorb efficiently analytes) are essential for the ISD efficiency of the matrix. Finally, due to their lower toxicity and their good stability (2-AA and 2-AB could be kept for several days in the dark without alteration of their properties) compared with the highly unstable 1,5-DAN matrix, these two compounds represent good alternatives for MALDI-ISD.

\section{Acknowledgments}

N.S. is an F.R.S.-F.N.R.S. logistic collaborator. The F.N.R.S. and Walloon Region contributed the mass spectrometry facility funding. Dr. Tyler A. Zimmerman is acknowledged for useful comments and additional editing of the manuscript.

\section{References}

1. Brown, R.S., Lennon, J.J.: Sequence-specific fragmentation of matrixassisted laser-desorbed protein/peptide ions. Anal. Chem. 67(21), 39903999 (1995)

2. Takayama, M.: In-source decay characteristics of peptides in matrixassisted laser desorption/ionization time-of-flight mass spectrometry. $J$. Am. Soc. Mass Spectrom. 12(4), 420-427 (2001)

3. Sakakura, M., Takayama, M.: In-source decay and fragmentation characteristics of peptides using 5-aminosalicylic acid as a matrix in matrix-assisted laser desorption/ionization mass spectrometry. J. Am. Soc. Mass Spectrom. 21, 979-988 (2010)

4. Demeure, K., Quinton, L., Gabelica, V., De Pauw, E.: Rational selection of the optimum MALDI matrix for top-down proteomics by in-source decay. Anal. Chem. 79(22), 8678-8685 (2007)

5. Resemann, A., Wunderlich, D., Rothbauer, U., Warscheid, B., Leonhardt, H., Fuchser, J., Kuhlmann, K., Suckau, D.: Top-down de novo protein sequencing of a $13.6 \mathrm{kDa}$ camelid single heavy chain antibody by matrix-assisted laser desorption ionization-time-of-flight/ time-of-flight mass spectrometry. Anal. Chem. 82(8), 3283-3292 (2010)

6. Suckau, D., Resemann, A.: T3-sequencing: targeted characterization of the $\mathrm{N}$ - and C-termini of undigested proteins by mass spectrometry. Anal. Chem. 75(21), 5817-5824 (2003)

7. Wuhrer, M., Deelder, A.M.: Matrix-assisted laser desorption/ionization in-source decay combined with tandem time-of-flight mass spectrometry of permethylated oligosaccharides: Targeted characterization of specific parts of the glycan structure. Rapid Commun. Mass Spectrom. 20(6), 943-951 (2006)

8. Yamagaki, T., Nakanishi, H.: Post-source decay fragmentation analyses of linkage isomers of Lewis-type oligosaccharides in curved-field reflectron matrix-assisted laser desorption/ionization time-of-flight mass spectrometry: Combined in-source decay/post-source decay experiments and relative ion abundance analysis. J. Mass Spectrom. 35(11), 1300-1307 (2000)

9. Yamagaki, T., Suzuki, H., Tachibana, K.: In-source and postsource decay in negative-ion matrix-assisted laser desorption/ionization timeof-flight mass spectrometry of neutral oligosaccharides. Anal. Chem. 77 (6), 1701-1707 (2005)

10. Bashir, S., Giannakopulos, A.E., Derrick, P.J., Critchley, P., Bottrill, A., Padley, H.J.: Matrix-assisted laser desorption/ionisation time-of-flight mass spectrometry. A comparison of fragmentation patterns of linear dextran obtained by in-source decay, post-source decay, and collisioninduced dissociation and the stability of linear and cyclic glucans studied by in-source decay. Eur. J. Mass Spectrom. (Chichester, Eng) 10(1), 109-120 (2004)

11. Kameyama, A., Kaneda, Y., Yamanaka, H., Yoshimine, H., Narimatsu, H., Shinohara, Y.: Detection of oligosaccharides labeled with cyanine dyes using matrix-assisted laser desorption/ionization mass spectrometry. Anal. Chem. 76(15), 4537-4542 (2004)
12. Smargiasso, N., De Pauw, E.: Optimization of matrix conditions for the control of MALDI in-source decay of permethylated glycans. Anal. Chem. 82(22), 9248-9253 (2010)

13. Wang, B.H., Hopkins, C.E., Belenky, A.B., Cohen, A.S.: Sequencing of modified oligonucleotides using in-source fragmentation and delayed pulsed ion extraction matrix-assisted laser desorption ionization time-offlight mass spectrometry. Int. J. Mass Spectrom. 169, 331-350 (1997)

14. Suckau, D., Resemann, A.: MALDI top-down sequencing: Calling Nand C-terminal protein sequences with high confidence and speed. $J$. Biomol. Tech. 20(5), 258-262 (2009)

15. Schulz, E., Karas, M., Rosu, F., Gabelica, V.: Influence of the matrix on analyte fragmentation in atmospheric pressure MALDI. J. Am. Soc. Mass Spectrom. 17(7), 1005-1013 (2006)

16. Gabelica, V., Schulz, E., Karas, M.: Internal energy build-up in matrixassisted laser desorption/ionization. J. Mass Spectrom 39(6), 579-593 (2004)

17. Demeure, K., Gabelica, V., De Pauw, E.A.: New advances in the understanding of the in-source decay fragmentation of peptides in MALDI-TOF-MS. J. Am. Soc. Mass Spectrom. 21(11), 1906-1917 (2010)

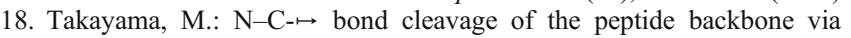
hydrogen abstraction. J. Am. Soc. Mass Spectrom. 12(9), 1044-1049 (2001)

19. Kocher, T., Engstrom, A., Zubarev, R.A.: Fragmentation of peptides in MALDI in-source decay mediated by hydrogen radicals. Anal. Chem. 77(1), 172-177 (2005)

20. Lennon, J.J., Walsh, K.A.: Locating and identifying posttranslational modifications by in-source decay during MALDI-TOF mass spectrometry. Protein Sci. 8(11), 2487-2493 (1999)

21. Netz, D.J., Pohl, R., Beck-Sickinger, A.G., Selmer, T., Pierik, A.J., Bastos Mdo, C., Sahl, H.G.: Biochemical characterisation and genetic analysis of aureocin A53, a new, atypical bacteriocin from Staphylococcus aureus. J. Mol. Biol. 319(3), 745-756 (2002)

22. Patterson, S.D., Katta, V.: Prompt fragmentation of disulfide-linked peptides during matrix-assisted laser desorption ionization mass spectrometry. Anal. Chem. 66(21), 3727-3732 (1994)

23. Quinton, L., Demeure, K., Dobson, R., Gilles, N., Gabelica, V., De Pauw, E.: New method for characterizing highly disulfide-bridged peptides in complex mixtures: Application to toxin identification from crude venoms. J. Proteome Res. 6(8), 3216-3223 (2007)

24. Schnaible, V., Wefing, S., Resemann, A., Suckau, D., Bucker, A., Wolf-Kummeth, S., Hoffmann, D.: Screening for disulfide bonds in proteins by MALDI in-source decay and LIFT-TOF/TOF-MS. Anal. Chem. 74(19), 4980-4988 (2002)

25. Deleuze, C., De Pauw, E., Quinton, L.: Selective reduction of $\mathrm{C}=\mathrm{C}$ double bonds in matrix-assisted laser desorption/ionization time-offlight mass spectrometry of microcystins. Eur. J. Mass Spectrom. (Chichester, Eng) 16(1), 91-99 (2010)

26. Fukuyama, Y., Iwamoto, S., Tanaka, K.: Rapid sequencing and disulfide mapping of peptides containing disulfide bonds by using 1,5-diaminonaphthalene as a reductive matrix. J. Mass Spectrom. 41(2), 191-201 (2006)

27. Asakawa, D., Takayama, M.: $\mathrm{C}(\mapsto)-\mathrm{C}$ bond cleavage of the peptide backbone in MALDI in-source decay using salicylic acid derivative matrices. J. Am. Soc. Mass Spectrom. 22(7), 1224-1233 (2011)

28. Takayama, M., Tsugita, A.: Sequence information of peptides and proteins with in-source decay in matrix assisted laser desorption/ ionization-time of flight-mass spectrometry. Electrophoresis 21(9), 1670-1677 (2000)

29. Zubarev, R.A., Kjeldsen, F., Haselmann, K.F., Budnik, B.A., Jensen, F.: Dissociative capture of hot $(3-13 \mathrm{eV})$ electrons by polypeptide polycations: An efficient process accompanied by secondary fragmentation. Chem. Phys. Lett. 356(3/4), 201-206 (2002)

30. Johnson, R.S., Martin, S.A., Biemann, K.: Collision-induced fragmentation of $(\mathrm{M}+\mathrm{H})+$ ions of peptides - side-chain specific sequence ions. Int. J. Mass Spectrom. Ion Processes 86, 137-154 (1988)

31. Diedrich, J. K., Julian, R. R. Facile identification of phosphorylation sites in peptides by radical directed dissociation. Anal. Chem. 83(17), 6818-6826 (2011)

32. Kruger, N.A., Zubarev, R.A., Horn, D.M., McLafferty, F.W.: Electron capture dissociation of multiply charged peptide cations. Int. J. Mass Spectrom. 187, 787-793 (1999) 